\title{
Multi-source Hierarchical Prediction Consolidation
}

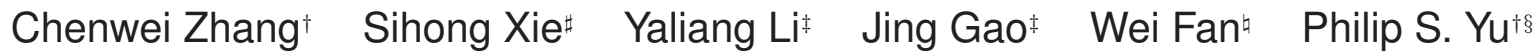 \\ †Department of Computer Science, University of Illinois at Chicago, Chicago, IL, USA \\ \#Computer Science and Engineering Department, Lehigh University, Bethlehem, PA, USA \\ ${ }^{\ddagger}$ SUNY Buffalo, Buffalo, NY, USA \\ ${ }^{\natural}$ Baidu Research Big Data Lab, Sunnyvale, CA, USA \\ $\S$ Institute for Data Science, Tsinghua University, Beijing, China \\ † czhang99,psyu\}@uic.edu, ${ }^{\sharp}$ sxie@cse.lehigh.edu, $¥\left\{\right.$ yaliangl,jing\}@buffalo.edu, ${ }^{\prime}$ fanwei03@baidu.com
}

\begin{abstract}
In big data applications such as healthcare data mining, due to privacy concerns, it is necessary to collect predictions from multiple information sources for the same instance, with raw features being discarded or withheld when aggregating multiple predictions. Besides, crowd-sourced labels need to be aggregated to estimate the ground truth of the data. Due to the imperfection caused by predictive models or human crowdsourcing workers, noisy and conflicting information is ubiquitous and inevitable. Although state-ofthe-art aggregation methods have been proposed to handle label spaces with flat structures, as the label space is becoming more and more complicated, aggregation under a label hierarchical structure becomes necessary but has been largely ignored. These label hierarchies can be quite informative as they are usually created by domain experts to make sense of highly complex label correlations such as protein functionality interactions or disease relationships.

We propose a novel multi-source hierarchical prediction consolidation method to effectively exploits the complicated hierarchical label structures to resolve the noisy and conflicting information that inherently originates from multiple imperfect sources. We formulate the problem as an optimization problem with a closed-form solution. The consolidation result is inferred in a totally unsupervised, iterative fashion. Experimental results on both synthetic and real-world data sets show the effectiveness of the proposed method over existing alternatives.
\end{abstract}

\section{Keywords}

Ensemble; Unsupervised Learning; Hierarchy; Crowdsourcing

\section{INTRODUCTION}

For various tasks such as crowdsourcing, healthcare data mining in big data applications, multiple information sources may provide labeling information on the same instance si-

Permission to make digital or hard copies of all or part of this work for personal or classroom use is granted without fee provided that copies are not made or distributed for profit or commercial advantage and that copies bear this notice and the full citation on the first page. Copyrights for components of this work owned by others than ACM must be honored. Abstracting with credit is permitted. To copy otherwise, or republish, to post on servers or to redistribute to lists, requires prior specific permission and/or a fee. Request permissions from permissions@acm.org.

CIKM'16, October 24-28, 2016, Indianapolis, IN, USA

(c) 2016 ACM. ISBN 978-1-4503-4073-1/16/10 . \$ $\$ 15.00$

DOI: http://dx.doi.org/10.1145/2983323.2983676 multaneously. Once we obtained the labeling information from multiple information sources or human beings, it is necessary to consolidate the collected information to infer the ground truth labels. Since the imperfect information from a single information source exists ubiquitously, it is also important that labeling information from multiple sources need to be consolidated to resolve noises and conflicts. Moreover, due to privacy concerns, raw features of instances are often discarded or withheld and only labels are available for aggregation purposes. For example in online healthcare forums, the raw features of a patient need to be discarded for privacy concerns and only diseases names collected from multiple doctors are consolidated to infer the ground truth.

In those applications, instead of assigning a single label for each instance, it is usually more informative to associate an instance with more than one labels to characterize multiple properties an instance has. For example, an image instance can be described by multiple tags such as "monarda", "bird" and hence belongs to multiple categories. A protein can be associated with more than one functions, denoting various functionalities. A patient may be associated with several candidate diseases, each of them diagnosed by a doctor.

Typically, those tasks consider all the labels on the same "flat" level. However, it is still insufficient to measure the value of the informativeness of labels when we isolate labels with each other and ignore the correlations between them. A better way is to organize labels in a hierarchical taxonomy. In this way, besides correlations such as co-occurrences among "flat" labels, a label hierarchy contains rich information to make sense of highly complex label correlations.

Problem Studied: In this paper, we want to incorporate the label hierarchy into the prediction consolidation process when only the labeling information from multiple information sources are available, which is formally defined as the Multi-source Hierarchical Prediction Consolidation(MHPC) problem, illustrated in Figure 1.

Informative label hierarchies are prevalently observed in various applications. For example, in crowdsourcing for protein functionality annotation, the functional labels of a protein are in a hierarchy, representing the functional relations. In healthcare data mining, disease labels can be also constructed with a tree-like disease taxonomy, denoting the pathology structure of human diseases. Current works in the literature $[1,2]$ try to consolidate predictions from multiple information sources. However, those works have access to raw features of the data and they totally ignore the hierarchical information, which may lead to potential loss of valuable information [3]. 


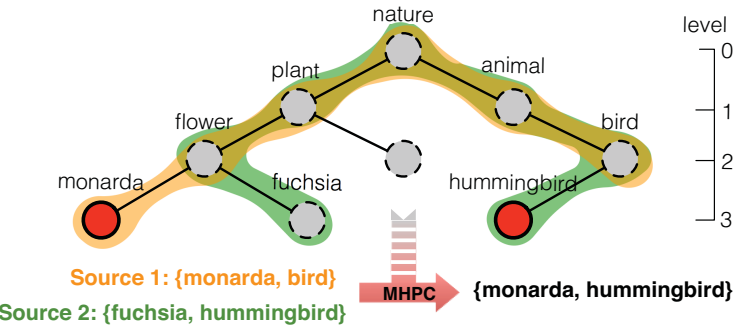

Figure 1: An illustrative example of the multi-source hierarchical prediction consolidation problem. Two individual information sources give label predictions to an image and MHPC tries to find a consolidated label prediction that maximizes the consensus among these predictions while preserving the structures of the label hierarchy.

With a number of approaches proposed to exploit the label hierarchy for various tasks [4] such as classification or clustering, it is reasonable to believe that informative label hierarchies, which inherently come with many prediction consolidation tasks, are also able to offer auxiliary and valuable information for the MHPC problem. For example, the conflicts between two label predictions in Figure 1 can be resolved by mapping label predictions to level 2, where we have $\{$ flower, bird $\}$ for both label predictions. Moreover, the label hierarchy may provide some constraints so that label predictions which violate the hierarchical structure will be less useful.

Given the importance of incorporating the label hierarchy, the MHPC problem itself is a novel problem which is rarely studied. Various learning problems are summarized in Figure 2, where model-level ensemble learning [5, 6, 7] tries to aggregate labels at the output level and hierarchical multi-label learning exploits the label hierarchy to improve the model performance on a wide range of multi-label learning tasks $[3,4]$. The multi-source hierarchical prediction consolidation problem is an unsupervised ensemble learning problem that aggregates hierarchical multi-label predictions on the model-level, where very few work has been done.

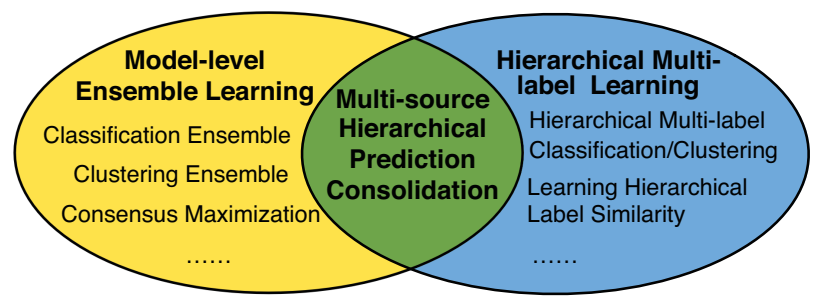

Figure 2: Position of the multi-source hierarchical prediction consolidation problem.

In this paper, we try to solve the MHPC problem by formulating it as an optimization task. The objective function for optimization favors the smoothness over all information sources as well as penalizing any two instances which have high hierarchical instance similarity but conflict with each other in the consolidation result. We derive a closed-form solution for this optimization problem. After that, the МРнC algorithm is introduced where two phases, namely estimat- ing hierarchical similarities and minimizing consensus cost, are conducted in an iterative, totally unsupervised fashion to get the consolidated label prediction for each instance.

\section{PROBLEM STATEMENT}

Before introducing the proposed method, we will give the definitions of some important concepts and formulation of the MHPC problem first in this section.

\subsection{Terminology Definition}

Suppose we are given label matrices $\mathbf{Y}_{1}, \mathbf{Y}_{2}, \ldots, \mathbf{Y}_{M}$ provided by $M$ information sources and a hierarchical adjacency matrix $\mathbf{H}$. An label matrix $\mathbf{Y}_{m}$ is an $N$ by $K$ matrix indicating the labeling information on all $N$ instances and $K$ labels provided by the information source $m$. Within $\mathbf{Y}_{m}$, the $i$-th row $\mathbf{Y}_{m(i)}$ is a label vector of instance $i$ provided by information source $m$, where $\mathbf{Y}_{m(i)}^{k}=1$ if the information source $m$ associates the instance $i$ with label $k$. A hierarchical adjacency matrix $\mathbf{H}$ embodies the hierarchical structure for all $K$ labels where $\mathbf{H}_{k k^{\prime}}=1$ if and only if when label $k$ is the direct descendant of label $k^{\prime}$. Other terminologies are introduced further when they are used.

\subsection{Problem Statement}

Based on the terminologies defined above, the Multisource Hierarchical Prediction Consolidation problem is formally defined as: given label matrices $\left\{\mathbf{Y}_{1}, \mathbf{Y}_{2}, \ldots, \mathbf{Y}_{m}\right\}$ and a hierarchical adjacency matrix $\mathbf{H}$, the MHPC problem tries to incorporate the label hierarchy into finding a consolidated label matrix $\hat{\mathbf{Y}}$ that maximizes the consensus among all label matrices. The maximum consensus is achieved by minimizing the consensus cost in prediction consolidation.

\section{MODELING HIERARCHICAL CONSEN- SUS}

This section describes how we come up with a consolidation agreement from multiple information sources by incorporating the label hierarchy into the minimization of consensus cost. Section 3.1 describes the objective function for minimizing the consensus cost as well as its closed-form solution. Section 3.2 integrates the hierarchical information into the objective function when estimating instance similarities.

\subsection{Minimizing the Consensus Cost}

We formulate the following objective function:

$$
\min _{\hat{\mathbf{Y}}} \frac{1}{M} \sum_{m=1}^{M}\left\|\hat{\mathbf{Y}}-\mathbf{Y}_{m}\right\|_{F}^{2}+\lambda \sum_{i=1}^{N} \sum_{j=1}^{N} \mathbf{W}_{i j}\left\|\hat{\mathbf{Y}}_{(i)}-\hat{\mathbf{Y}}_{(j)}\right\|_{2}^{2} \quad \text { s.t. } \quad \lambda \geqslant 0,
$$

where $\|$.$\| denotes the \ell 2$-norm for matrices and vectors. The first term favors the smoothness of the consolidation result over label predictions from all the information sources. The second term serves as a regularization term which ensures that label vectors of any two instances in the consolidation result(the $i$-th and the $j$-th instance in $\hat{\mathbf{Y}}$ ) do not differentiate themselves from each other very much if they share a high hierarchical instance similarity $\mathbf{W}_{i j}$. Estimation for $\mathbf{W}$ will be further explained in Section 3.2. $\lambda$ serves as a regularization coefficient to penalize violation for the hierarchical similarity constraints. 
Note that the objective function in Equation 1 can be rewritten in a matrix form as:

$$
\begin{aligned}
\mathrm{J}(\hat{\mathbf{Y}}) & =\frac{1}{M} \sum_{m=1}^{M}\left(\operatorname{Tr}\left(\hat{\mathbf{Y}}^{\mathrm{T}} \hat{\mathbf{Y}}\right)-2 \operatorname{Tr}\left(\mathbf{Y}_{m}{ }^{\mathrm{T}} \hat{\mathbf{Y}}\right)+\operatorname{Tr}\left(\mathbf{Y}_{m}{ }^{\mathrm{T}} \mathbf{Y}_{m}\right)\right) \\
& +\lambda \operatorname{Tr}\left(\hat{\mathbf{Y}}^{\mathrm{T}} \mathbf{L} \hat{\mathbf{Y}}\right) \quad \text { s.t. } \quad \lambda \geqslant 0
\end{aligned}
$$

where $\mathbf{L}$ is the symmetric normalized Laplacian matrix

$$
\mathbf{L}=\mathbf{D}^{-\frac{1}{2}}(\mathbf{D}-\mathbf{W}) \mathbf{D}^{-\frac{1}{2}}
$$

and $\mathbf{D}$ is the degree matrix of $\mathbf{W}$.

To find a $\hat{\mathbf{Y}}$ that gives the minimum value of $\mathrm{J}(\hat{\mathbf{Y}})$, we first prove the convexity of $\mathrm{J}(\hat{\mathbf{Y}})$ by showing the positive definite property of the Hessian matrix of $J(\hat{\mathbf{Y}})$ with respect to $\hat{\mathbf{Y}}$.

$$
\frac{\partial^{2} \mathrm{~J}(\hat{\mathbf{Y}})}{\partial \hat{\mathbf{Y}}^{2}}=2(\mathbf{I}+\lambda \mathbf{L}) \quad \text { s.t. } \quad \lambda \geqslant 0 .
$$

The Hessian matrix shown in Equation 4 is positive definite because $\lambda \mathbf{L}$ is positive-semidefinite [8] and adding an identity matrix to it makes the resulting Hessian matrix positive definite [9]. Therefore, setting the derivative of $\mathrm{J}(\hat{\mathbf{Y}})$ with respect to $\hat{\mathbf{Y}}$ to zero

$$
\frac{\partial \mathrm{J}(\hat{\mathbf{Y}})}{\partial \hat{\mathbf{Y}}}=\frac{1}{M} \sum_{m=1}^{M}\left(2 \hat{\mathbf{Y}}-2 \mathbf{Y}_{m}\right)+2 \lambda \mathbf{L} \hat{\mathbf{Y}}=0
$$

leads to a global minimized consensus cost $\mathrm{J}(\hat{\mathbf{Y}})$ given $\mathbf{W}$.

A closed-form solution can be obtained by solving the Equation 5:

$$
\begin{aligned}
\hat{\mathbf{Y}} & =\left(1+\lambda\left(\mathbf{D}^{-\frac{1}{2}}(\mathbf{D}-\mathbf{W}) \mathbf{D}^{-\frac{1}{2}}\right)\right)^{-1} \frac{1}{M} \sum_{m=1}^{M} \mathbf{Y}_{m} \\
& =(\mathbf{I}+\lambda \cdot \mathbf{L}))^{-1} \overline{\mathbf{Y}}
\end{aligned}
$$

where $\overline{\mathbf{Y}}=\frac{1}{M} \sum_{m=1}^{M} \mathbf{Y}_{m}$ and $\mathbf{I}$ is the identity matrix.

From Equation 6, we can see that hierarchical similarity constraints are not introduced when $\lambda=0$. In this case, the consolidation result degrades to the simple averaging of all the label prediction we obtained. While $\lambda>0$, the Laplacian matrix $\mathbf{L}$ regularizes the simple averaging result and guides the $\overline{\mathbf{Y}}$ towards a global consensus $\hat{\mathbf{Y}}$ with label hierarchies being considered.

\subsection{Estimating the Hierarchical Similarity}

Given any two label vectors, each of which denotes a label prediction for an instance, an instance similarity matrix $\mathbf{W}$ in Equation 1 is inferred from the consolidation result to measure the similarities between label vectors. We assume that each label has a unique degree of support, asserting that such label belongs to an instance. Then, the instance similarity value $\mathbf{W}_{i j}$ of any two instance $i$ and instance $j$ can be calculated by the following Equation:

$$
\mathbf{W}_{i j}=\exp \left(-\frac{1}{\sigma} \sqrt{\sum_{k=1}^{K} \vec{S}_{k}\left(\hat{\mathbf{Y}}_{(i)}^{k}-\hat{\mathbf{Y}}_{(j)}^{k}\right)^{2}}\right)
$$

where $\sqrt{\sum_{k=1}^{K} \vec{S}_{k}\left(\hat{\mathbf{Y}}_{(i)}^{k}-\hat{\mathbf{Y}}_{(j)}^{k}\right)^{2}}$ in Equation 7 measures the weighted Euclidean distance between label vectors of two consolidated instances in $\hat{\mathbf{Y}}$, namely $\hat{\mathbf{Y}}_{(i)}$ and $\hat{\mathbf{Y}}_{(j)} \cdot \vec{S}$ is a support label vector that serves as weights for distance measurement where each entry $\vec{S}_{k}$ of $\vec{S}$ indicates the degree of support of the label $k$ to the distance estimation. $\sigma$ is a constant factor and the exponential function $\exp ($.$) converts$ the distance measurement to a similarity measurement.

Usually, we assume that each label has an individual degree of support to the overall similarity estimation. However, as the label space is becoming more and more complicated, such simplified assumption totally ignores correlations among labels and therefore will lead to an inaccurate similarity estimation due to the label sparsity. For example, there can be thousands of labels for flower names such as "monarda", "fuchsia" and so on. However, it is very unlikely that label predictions provided by various information sources cover all of those flower names. Although these labels share the same general idea (the "flower"), if the support value is calculated on each label separately, none of those labels is able to contribute to the support value of label "flower" which they share the general idea with. Also, different information sources may have preferences in providing certain labels so remaining labels may be rarely used.

The label hierarchy organizes labels in a tree-like structure in which general labels are the ancestors of specific labels. With a label hierarchy being incorporated, labels are no longer independent of each other: the general idea is that when an information source assigns the label $k$ to an instance, the existence of that label reflects a direct occurrence of this label to support the instance. Moreover, such label assignment on label $k$ also indicates indirect occurrences, although not explicitly labeled, from its ancestor labels on the label hierarchy. Therefore, we would like to let the occurrence of each label contributes not only to itself but also to all its ancestor labels in a label hierarchy.

To make this happen, we first apply the label augmentation algorithm to convert each label vector $\mathbf{Y}_{\mathbf{m}(i)}$ to an augmented label vector $\mathbf{Y}_{m(i)}^{*}$, as shown in Algorithm 1.

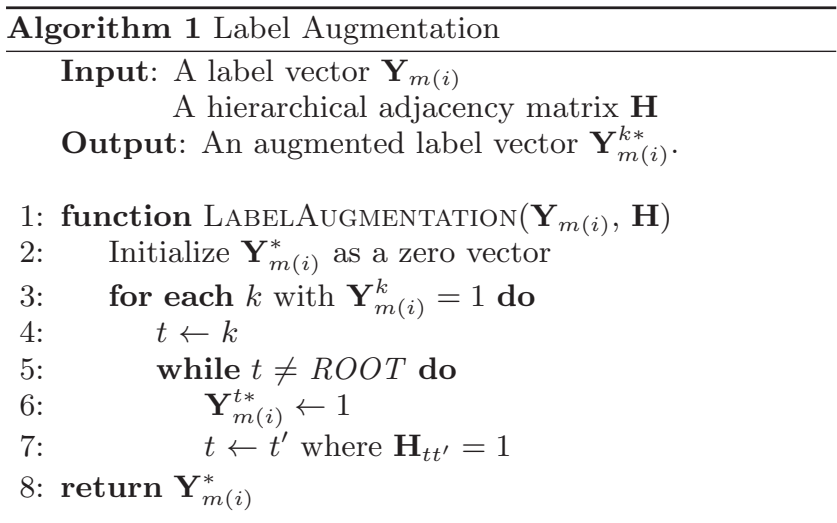

By augmentation, the direct occurrence of a label in the label vector contributes not only to itself, but also to its ancestor labels in the hierarchy. We propose to use the occurrence value $\vec{C}_{k}=\sum_{m=1}^{M} \sum_{i=1}^{N} \mathbf{Y}_{m(i)}^{k *}$ of a label $k$ in all augmented label vectors to estimate the support value for each label.

A label $k$ which is near the root node in a label hierarchy tends to have a higher occurrence $\vec{C}_{k}$ value in augmented label vectors. Asserting the belongingness of a label which is in proximity to the root node to an instance is less likely to be fallible. Therefore, labels with high occurrence values make themselves more supportive in similarity estimation. By summing up the occurrence of each label in all the aug- 
mented label vectors, labels close to the root node in the hierarchy are frequently augmented, thus have higher occurrence values $\vec{C}_{k}$.

However, using the occurrence value to estimate the support value can be very inaccurate because although the label predictions are collected from multiple information sources, the occurrences of many labels near the leaf nodes in a label hierarchy only share a very small portion over the sum of occurrences, even after label augmentation. This leads to very small occurrence values for all those labels.

In this work, we model the occurrence of a label in an augmented label vector with a confidence interval. The occurrence of a label within all augmented label vectors can be considered as it is sampled from a subset of a population of labels. The occurrence information will be more accurate when we observe this label more frequently among all the augmented label vectors, which leads to a narrow confidence bound. Otherwise, if we rarely observe the occurrence of a label, the resulting confidence bound will be wide and it will be riskier to incorporate this piece of occurrence information into the support value calculation. By incorporating confidence intervals, the occurrence value itself shows how uncertain we are about the occurrence value of a label.

We use the occurrence value $\vec{C}_{k}$ of label $k$ over the number of instances $N$ as the proportion. Since the root node will be activated in all the augmented label vectors, the root node will have the highest proportion as 1 . One label is either activated or not in an augmented label vector, therefore the binomial probability distribution is used. The resulting confidence interval can be presented in an alternate formulation that uses quantiles from the beta distribution [10]:

$$
B\left(\frac{\alpha}{2} ; \vec{C}_{k}, N-\vec{C}_{k}+1\right)<\theta_{k}<B\left(1-\frac{\alpha}{2} ; \vec{C}_{k}, N-\vec{C}_{k}+1\right),
$$

where $B$ is the Beta distribution and $\alpha$ is the significance level, which usually has the value $0.05(5 \%)$. $\theta_{k}$ is the probability of a label $k$ being activated in an augmented label vector. $\vec{C}_{k}$ is the occurrence value of label $k$ over all augmented label vectors.

The Equation 8 has an appealing property that the less frequent a label $k$ being sampled, the wider confidence interval it will end up with. A narrow confidence bound indicates a stronger certainty during the support value calculation. Therefore the lower bound value of the confidence interval is used to calculate the support value for each label:

$$
\vec{S}_{k}=B\left(\frac{\alpha}{2} ; \vec{C}_{k}, N-\vec{C}_{k}+1\right)
$$

Once we calculate the support value for each label with confidence, the instance similarity matrix $\mathbf{W}$ in Equation 7 can be calculated with the support label vector $\vec{S}$ learned from the label hierarchy. Hence, $\mathbf{W}$ is called a hierarchical instance similarity matrix.

\section{CONSOLIDATING HIERARCHICAL LABELS}

\subsection{The MнPс Algorithm}

Although we provide a closed-form solution to find the global minimum for the consensus cost when the hierarchical instance similarity matrix $\mathbf{W}$ is given, it is still hard to estimate both $\hat{\mathbf{Y}}$ that associates with a minimized consensus $\operatorname{cost} J(\hat{\mathbf{Y}})$ and the hierarchical instance similarity matrix $\mathbf{W}$ at the same time. Hence, we propose a two-phase iterative algorithm, namely the multi-source hierarchical prediction consolidation (MHPC) algorithm, which naturally decouples the computation within each iteration.

The MHPC algorithm has two phases within each iteration: estimating the hierarchical similarity (Section 3.2) and minimizing the consensus cost (Section 3.1). The MHPC algorithm starts at iteration $t_{0}$ with an initial estimation for hierarchical instance similarity matrix $\left(\mathbf{W}_{i j}\right)_{t_{0}}$ and the consolidation result $(\hat{\mathbf{Y}})_{t_{0}} \cdot\left(\mathbf{W}_{i j}\right)_{t_{0}}$ is initialized by the following equation:

$$
\left(\mathbf{W}_{i j}\right)_{t_{0}}=\exp \left(-\frac{1}{\sigma} \sqrt{\sum_{k=1}^{K} \vec{S}_{k}\left(\overline{\mathbf{Y}}_{(i)}^{k}-\overline{\mathbf{Y}}_{(j)}^{k}\right)^{2}}\right)
$$

where $\overline{\mathbf{Y}}_{(i)}^{k}$ is the simple averaging result on the $k$-th label of the instance $i$ from all $M$ sources, calculated by $\overline{\mathbf{Y}}_{(i)}^{k}=$ $\frac{1}{M} \sum_{m=1}^{M} \mathbf{Y}_{m(i)}^{k}$. Note that support values in $\vec{S}$ and the occurrence values in $\vec{C}$ are derived from all the multi-source label predictions we obtained, which we only initialize once in the entire algorithm, regardless of iterations. $(\hat{\mathbf{Y}})_{t_{0}}$ is initialized using the Equation 6.

Once we obtain an initial value for $\left(\mathbf{W}_{i j}\right)_{t_{0}}$ and $(\hat{\mathbf{Y}})_{t_{0}}$, each iteration afterwards follows the following updating rules. Estimating the hierarchical similarity:

$$
\left(\mathbf{W}_{i j}\right)_{t_{x+1}}=\exp \left(-\frac{1}{\sigma} \sqrt{\sum_{k=1}^{K} \vec{S}_{k}\left(\left(\hat{\mathbf{Y}}_{(i)}^{k}\right)_{t_{x}}-\left(\hat{\mathbf{Y}}_{(j)}^{k}\right)_{t_{x}}\right)^{2}}\right)
$$

where the hierarchical instance similarity $\left(\mathbf{W}_{i j}\right)_{t_{x+1}}$ is calculated by the most up-to-date consolidation result $(\hat{\mathbf{Y}})_{t_{\mathrm{x}}}$. Minimizing the consensus cost:

$$
(\hat{\mathbf{Y}})_{t_{x+1}}=\left(1+\lambda \cdot(\mathbf{L})_{t_{\mathrm{x}}}\right)^{-1}(\hat{\mathbf{Y}})_{t_{\mathrm{x}}}
$$

where the laplacian matrix $(\mathbf{L})_{t_{\mathrm{x}}}$ is calculated by the most up-to-date $(\mathbf{W})_{t_{x}}$ value using Equation 3 . Note that in Equation 12, the consolidation result in the latest iteration $(\hat{\mathbf{Y}})_{t_{\mathrm{x}}}$ is used for updating, rather than $\overline{\mathbf{Y}}$ as shown in Equation 6 . With this updating function, the consolidation result can accumulate the consolidation progresses from previous iterations. Otherwise, if $(\overline{\mathbf{Y}})_{t_{x}}$ is used in Equation 12, we simply ignore the consolidation results from all the previous iterations, and $\mathbf{L}_{t_{x}}$ is the only factor we can rely on to guide the consolidation process toward a final consensus. The algorithm terminates whenever the updates on the consolidation result $(\hat{\mathbf{Y}})_{t_{x+1}}$ is no longer significant after an iteration.

\section{EXPERIMENTS}

In this section, the yeast data sets, the real-world medical data sets and their label hierarchies are briefly introduced. After that, the performance of the MHPC algorithm is compared with other alternatives on both data sets. Experiments on yeast data sets illustrate the ability of the proposed method in overcoming the various degree of label vagueness and noise from multiple information sources. While the real-world medical data set emphasizes more on the label sparsity because in real-world medical consultation, we can't ensure that all the information sources provide labels to all instances. Due to page limitation, experiment details such as detailed data description, convergence analysis on the proposed algorithm as well as parameter estimation can be found in [11]. 


\subsection{Data description and data preprocessing}

\subsubsection{Yeast Data Sets}

The yeast data sets has ten subsets, each annotates yeast genome from different aspects. Each yeast genome is annotated with hierarchical-structured labels in the Functional Catalogue (FunCat) [12]. For example, a yeast genome can be associated with three functionalities: $\{20 / 01 / 03 / 01$ (sugar transport), 20/03/02/02/01 (proton driven symporter), 20/09/18 (cellular import)\}. The annotation scheme follows the protein functional description of each genome instance, with up to 6 levels of label taxonomy. On average, each instance has 8.8 labels.

Based on the ground truth multi-label label predictions, we introduce vague labels, as well as noisy labels to the yeast data sets to model real-world cases where imperfect predictive models or inexperienced human annotators are involved in MHPC problems. The algorithm for generating the synthetic datasets can be found in [11].

\subsubsection{Medical Data Sets}

The medical data set and the disease label hierarchy are obtained from an online medical consultation website xywy.com ${ }^{1}$, where patients post their healthcare related questions and multiple medical professionals give online suggestions or general advice as answers.

Given certain symptoms, each doctor is considered as an individual information source that provides disease names as labels. Ground truth disease labels are obtained by a medical knowledge base in Baidu Baike ${ }^{2}$ (an online encyclopedia of Baidu) where registered doctors provide knowledge about certain disease names that closely associate with some symptoms. The disease label hierarchy is organized in an anatomical structure, with up to three levels of labels(e.g. disease - otorhinolaryngology - rhinitis). Usually, a doctor provides around 2.5 labels to an instance on average, which leads to a low label coverage over all labels in a hierarchy: not all doctors provide labels for each instance; not all instances get label predictions from every doctor. Only $0.011506 \%$ of labels are activated among label predictions over all instances from all the information sources we obtained.

\subsection{Comparison Methods}

To show the advantages of the MHPC algorithm in solving multi-source hierarchical prediction consolidation problem, we compare the MHPC method with many baseline methods. Considering that no known multi-source hierarchical prediction consolidation methods are available, averaging methods as well as other model-level ensemble learning methods are introduced, which can be divided into three categories:

Averaging Methods

- SA: The simple averaging method. The SA method simply takes the average of multi-source label predictions. In [13], the authors observe that the simple averaging method is competitive with a variety of adaptive algorithms under the quadratic loss criterion.

- WA: The weighted averaging method. Besides the simple averaging method which considers an equal contribution of each label to the consolidation result, the WA weights labels by support values learned in Section 3.2.

\footnotetext{
${ }^{1}$ http://club.xyxy.com

${ }^{2}$ http://baike.baidu.com
}

\section{Consensus Maximization Methods}

- MLCM: The multi-label consensus maximization method is introduced in [6]. The MLCM learns a consolidation result from both label predictions and cluster predictions of the same instance from multiple information sources. By ignoring the cluster predictions, the MLCM adapts to the problem setting of MHPC. However, the label hierarchy is not explored in MLCM.

Multi-source Prediction Consolidation Methods - MPC-U: The multi-source prediction aggregation method that minimizes the consensus cost as mentioned in Section 3.1 , but uses a uniform support label vector $\vec{S}$ during instance similarity estimation.

- Mhpc: The proposed method which minimizes the consensus cost by optimization and incorporates the label hierarchy in estimating hierarchical instance similarities. The support value of each label is estimated based on the lower bound confidence interval of the proportion of occurrence based on all the augmented label vectors we obtained.

\subsection{Experimental results}

\subsubsection{Yeast Data Sets}

For yeast data sets, two parameters $\left(\alpha_{\text {vague }}\right.$ on $\mathrm{x}$-axis and $\alpha_{\text {noise }}$ on y-axis) are varied from 0.1 to 1 to test how sensitive vague and noisy labels will affect the model performance. The performance on three evaluation metrics (Ranking loss, micro-AUC [14] and coverage error [15] on z-axes) of the MHPC method and other alternatives are compared on each combination of $\alpha_{\text {vague }}$ and $\alpha_{\text {noise }}$. For each model, the performance on all combinations of $\alpha_{\text {vague }}$ and $\alpha_{\text {noise }}$ values are connected as a surface. Due to space limitation, only the result on church, one of the ten yeast data sets, is reported with $\lambda=10$. Multi-source label predictions from four information sources are used.
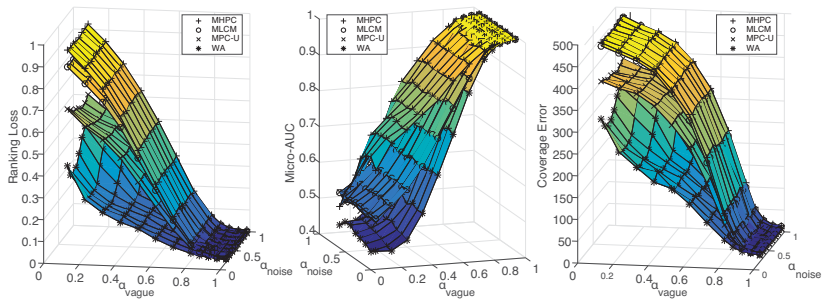

Figure 3: Performance with varying degrees of vagueness and noises on church, one of the yeast data sets.

Figure 3 shows the performance comparison results. We observe that the MPHC (the surface with the "+" sign) outperforms other alternatives consistently. When the vagueness level $\alpha_{\text {noise }}$ increases, the performance deteriorates for all the methods. However, the MHPC performs relatively better than others. On the other hand, when the noise level increases and $\alpha_{\text {vague }}$ is fixed, WA and MPC-U methods are more easily affected by the noisy label, which leads to fluctuations on the performance surfaces. While MHPC has a relative stable performance when we varies $\alpha_{\text {noise }}$ from 0.1 to 1 on almost all the values $\alpha_{\text {vauge }}$ can take. Note that the performance of $\mathrm{SA}$ is similar with WA, so the performance of $\mathrm{SA}$ is not presented in this figure. 


\subsubsection{Medical Data Sets}

The medical data sets come with multi-source label predictions inherently when multiple doctors gives suggestions to each patient. The medical date sets consist of six subsets (MED.1 to MED.6) and we randomly sampled 500 instances from each data set. The label predictions associate with those instances are used for prediction consolidation. Table 1 shows the performance of MHPC with other baseline methods, from which we can see the superior performance of MHPC method in real-world scenarios.

\begin{tabular}{|c|c|c|c|c|}
\hline \multirow{2}{*}{ Datasets } & \multirow{2}{*}{ Methods } & \multicolumn{3}{|c|}{ Evaluation Metrics } \\
\hline & & Ranking Loss & Micro-AUC & Coverage Error \\
\hline \multirow{5}{*}{ MED.1 } & $\mathrm{SA}$ & $0.520(5)$ & $0.620(5)$ & $213.175(5)$ \\
\hline & WA & $0.518(4)$ & $0.621(3)$ & $212.844(4)$ \\
\hline & MPC-U & $0.304(3)$ & $0.547(5)$ & $125.108(3)$ \\
\hline & MLCM & $0.262(2)$ & $0.643(2)$ & $108.146(1)$ \\
\hline & МнРс & $0.196(1)$ & $0.754(1)$ & $125.107(2)$ \\
\hline \multirow{5}{*}{ MED.2 } & $\mathrm{SA}$ & $0.358(5)$ & $0.603(4)$ & $75.544(5)$ \\
\hline & WA & $0.357(4)$ & $0.604(3)$ & $75.416(4)$ \\
\hline & MPC-U & $0.200(2)$ & $0.539(5)$ & $42.630(3)$ \\
\hline & MLCM & $0.268(3)$ & $0.680(2)$ & $31.221(1)$ \\
\hline & МнРс & $0.166(1)$ & $0.715(1)$ & $35.336(2)$ \\
\hline \multirow{5}{*}{ MED.3 } & $\mathrm{SA}$ & $0.067(4)$ & $0.706(2)$ & $3.400(4)$ \\
\hline & WA & $0.065(3)$ & $0.704(3)$ & $3.314(3)$ \\
\hline & MPC-U & $0.069(5)$ & $0.432(5)$ & $3.543(5)$ \\
\hline & MLCM & $0.064(2)$ & $0.542(4)$ & $3.288(2)$ \\
\hline & МнPC & $0.038(1)$ & $0.847(1)$ & $2.000(1)$ \\
\hline \multirow{5}{*}{ MED.4 } & $\mathrm{SA}$ & $0.301(5)$ & $0.601(4)$ & $38.325(5)$ \\
\hline & WA & $0.300(4)$ & $0.602(3)$ & $38.275(4)$ \\
\hline & MPC-U & $0.173(2)$ & $0.460(5)$ & $22.242(3)$ \\
\hline & MLCM & $0.225(3)$ & $0.617(2)$ & $19.325(2)$ \\
\hline & МнPC & $0.118(1)$ & $0.707(1)$ & $15.233(1)$ \\
\hline \multirow{5}{*}{ MED. 5} & $\mathrm{SA}_{\mathrm{A}}$ & $0.355(5)$ & $0.563(4)$ & $67.534(5)$ \\
\hline & WA & $0.353(4)$ & $0.564(3)$ & $67.170(4)$ \\
\hline & MPC-U & 0.189 (1) & $0.549(5)$ & $36.080(2)$ \\
\hline & MLCM & $0.276(3)$ & $0.565(2)$ & $52.523(3)$ \\
\hline & МнPC & $0.232(2)$ & $0.572(1)$ & $32.648(1)$ \\
\hline \multirow{5}{*}{ MED.6 } & $\mathrm{SA}$ & $0.365(5)$ & $0.598(3)$ & $47.175(5)$ \\
\hline & WA & $0.363(4)$ & $0.599(2)$ & $46.991(4)$ \\
\hline & MPC-U & $0.236(3)$ & $0.491(5)$ & $30.632(3)$ \\
\hline & MLCM & $0.190(1)$ & $0.632(1)$ & $24.798(2)$ \\
\hline & МнPC & $0.203(2)$ & $0.594(4)$ & $22.633(1)$ \\
\hline
\end{tabular}

Table 1: Performance on medical data sets.

\section{CONCLUSIONS}

As information explodes, we are able to obtain an increasing number of label predictions from a large population of information sources at the same time. Due to privacy concerns or storage limitations, the raw features of instances are usually discarded or withheld. The labels we collect from multiple information sources bring not only diversity of labels, but also vagueness and noises. In this work, we studied the multi-source hierarchical prediction consolidation (MHPC) problem. Traditional model-level ensemble learning problems deal with multi-source information but they simply ignore the hierarchical structure of labels. On the other hand, hierarchical multi-label learning problems try to bring the label hierarchy into varies single-source learning tasks such as classification. The MHPC problem effectively incorporates the existing label hierarchy to resolve vagueness and noise originate from multiple information sources, where very few work has been done. We formulate the MHPC problem as an optimization task with a closed-form solution. Two phases, namely minimizing the consensus cost and estimating the hierarchical similarity, are performed in an iterative fashion to learn a consolidation result while preserving the structures of the label hierarchy. Experiments conducted on both synthetic and real-world data sets show the advantages of the proposed method over other alternatives.

\section{ACKNOWLEDGMENTS}

This work is supported in part by NSF through grants III1526499. We would like to thank the anonymous reviewers for helpful comments. We gratefully acknowledge the support of NVIDIA Corporation with the donation of the Titan $\mathrm{X}$ GPU used for this research.

\section{REFERENCES}

[1] J Ross Quinlan. Bagging, boosting, and C4. 5. In AAAI/IAAI, Vol. 1, 1996.

[2] Chi-Hoon Lee. Learning to combine discriminative classifiers: confidence based. In KDD, 2010.

[3] Lijuan Cai and Thomas Hofmann. Hierarchical document categorization with support vector machines. In CIKM, pages 78-87, 2004.

[4] Wei Bi and James T Kwok. Mandatory leaf node prediction in hierarchical multilabel classification. In NIPS, pages 153-161, 2012.

[5] Jing Gao, Feng Liang, Wei Fan, Yizhou Sun, and Jiawei Han. Graph-based consensus maximization among multiple supervised and unsupervised models. In NIPS, pages 585-593. 2009.

[6] Sihong Xie, Xiangnan Kong, Jing Gao, Wei Fan, and Philip S. Yu. Multilabel consensus classification. In ICDM, pages 1241-1246, 2013.

[7] Bowen Dong, Sihong Xie, Jing Gao, Wei Fan, and Philip S. Yu. OnlineCM: Real-time consensus maximization with missing values. In SDM, 2014.

[8] Bojan Mohar, Y Alavi, G Chartrand, and OR Oellermann. The laplacian spectrum of graphs. Graph theory, combinatorics, and applications, 2(871-898):12, 1991.

[9] A Ravindran, Gintaras Victor Reklaitis, and Kenneth Martin Ragsdell. Engineering optimization: methods and applications. John Wiley \& Sons, 2006.

[10] Lawrence D Brown, T Tony Cai, and Anirban DasGupta. Interval estimation for a binomial proportion. Statistical science, pages 101-117, 2001.

[11] Chenwei Zhang, Sihong Xie, Yaliang Li, Jing Gao, Wei Fan, and Philip S. Yu. Multi-source Hierarchical Prediction Consolidation. ArXiv e-prints arXiv: 1608.03344, 2016.

[12] Andreas Ruepp, Alfred Zollner, Dieter Maier, Kaj Albermann, Jean Hani, Mokrejs, et al. The funcat, a functional annotation scheme for systematic classification of proteins from whole genomes. Nucleic acids research, 32(18):5539-5545, 2004.

[13] Varsha Dani, Omid Madani, David Pennock, and Sumit Sanghai. An empirical comparison of algorithms for aggregating expert predictions. In UAI, 2006.

[14] Corinna Cortes and Mehryar Mohri. Auc optimization vs. error rate minimization. NIPS, 16(16), 2004.

[15] Grigorios Tsoumakas, Ioannis Katakis, and Ioannis Vlahavas. Mining multi-label data. In Data mining and knowledge discovery handbook, pages 667-685. Springer, 2009. 